\title{
Wireless Measurement of Moisture Entry in SYLGARD-527
}

\author{
Kirsten J. Dehning, Moritz Hitzemann, Stefan Zimmermann \\ Leibniz University Hannover, Institute of Electrical Engineering and Measurement Technology, \\ Appelstr. 9A, 30167 Hannover, Germany, \\ dehning@geml.uni-hannover.de
}

\begin{abstract}
Summary:
Humidity highly affects the failure rate of silicone-potted frequency converters in wind turbines. In order to understand the entry process of moisture, in this work, possible pathways for humidity e.g. by diffusing through the silicone-potting compound or passing along boundaries are investigated by potted humidity sensors. To avoid the effect of cables as additional entry gateways and the diffusion through sidewalls humidity sensors were potted into silicone inside an open toped glass vessel at different positions, and inductively powered and read out via Bluetooth. The results show that under the experimental conditions the moisture mainly diffuses through the silicone and does not pass along the boundary layer between the silicone and the glass.
\end{abstract}

Keywords: silicone-potting compound, wind turbine converter failure rate, moisture

\section{Introduction}

Due to the high failure rate of frequency converters in wind turbines and the associated repair costs and loss of earnings, Germany suffers major economic damage in the region of 200 million euros per year. Various research projects have indicated that moisture is a major cause of failure. Especially the work of Harley et al. shows that water vapor is absorbed in silicone-potting compounds damaging the converter electronics [1]. Furthermore Zorn et al. determined the effect of humidity on failure rate of converters [2].

To investigate possible entry pathways of moisture and its transport in silicone-potting compounds used to seal and protect the semiconductor modules from the environment, humidity and temperature sensors from Sensirion (Switzerland, Model SHT35-DIS-B) are fully potted into silicone inside an open glass vessel at different positions. To avoid additional gateways for humidity by cables, in this work, both the energy supply and the data transmission are wireless by inductive powering and Bluetooth. A QI coil placed at the bottom of the vessel is used for inductive powering the potted electronics, while data are transferred from a potted Arduino to an external Arduino connected to a computer using the Bluetooth low energy protocol. Furthermore, the open toped vessel is made of glass to prevent moisture from penetrating from the side.

The used silicone-potting compound is the coldcuring two-component silicone SYLGARD-527 (Dow, USA), that is chemically known as polydimethylsiloxane and which is very similar to the silicones used in the semiconductor industry. The temperature and humidity sensor SHT35DIS-B has an accuracy tolerance of $\pm 1.5 \%$ r.H. [3]. Here, we use eight of such sensors at different positions inside the silicone-potting compound. Four sensors are placed in the middle at different heights and four sensors are placed at one height but with different distances to the sidewall. The different sensor positions inside the silicone-potting compound are given in Tab. 1. The internal Arduino and the energy supply coil are placed far below the sensors. The setup is shown in Fig. 1.

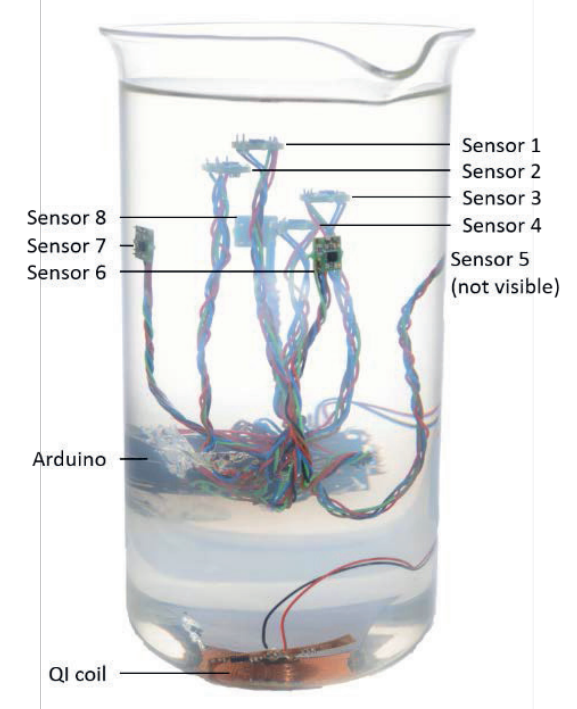

Fig. 1: Wireless measuring setup with eight sensors silicone-potted in an open toped vessel. 
Tab. 1: Sensor positions

\begin{tabular}{|c|c|c|}
\hline $\begin{array}{c}\text { Sensor } \\
\text { no. }\end{array}$ & $\begin{array}{c}\text { Distance from the } \\
\text { top of the silicone- } \\
\text { potting compound } \\
\text { [mm] }\end{array}$ & $\begin{array}{c}\text { Distance to } \\
\text { sidewall } \\
\text { [mm] }\end{array}$ \\
\hline 1 & 20 & 35 \\
\hline 2 & 30 & 35 \\
\hline 3 & 40 & 35 \\
\hline 4 & 50 & 35 \\
\hline 5 & 60 & 2 \\
\hline 6 & 60 & 7 \\
\hline 7 & 60 & 12 \\
\hline 8 & 60 & 17 \\
\hline
\end{tabular}

\section{Results}

In order to investigate the path of moisture through the silicone-potting compound a temporal step profile of relative humidity at constant temperature of $60^{\circ} \mathrm{C}$ was generated in a climatic chamber. The signals of all eight sensors are shown in Fig. 2. Obviously, the sensors 1 to 4 measure the rising moisture delayed according to the increasing distance from the top of the silicone potting. Even more important, the sensors 5 to 8 show similar response. This clearly indicates that the moisture does not pass along boundary layer between glass vessel wall and the silicone potting.

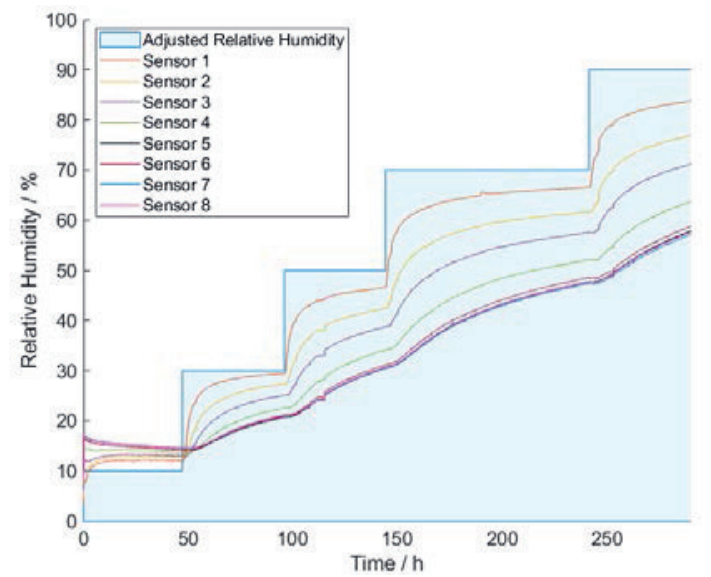

Fig. 2: Sensor signals of all eight silicone-potted humidity sensors responding to a temporal step profile of relative humidity at $60^{\circ} \mathrm{C}$.

Furthermore, we calculated the time constants of the sensor responses. Therefore, we used an exponential function fitted to the sensor responses 1 to 4 for the first step from $10 \%$ r.H. to $30 \%$ r.H. This is exemplarily shown in Fig. 3 for the first sensor. The time constants are given in Tab. 2. As expected, the second sensor has about twice the time constant compared to the first sensor although the distance to the surface is just $50 \%$ larger.

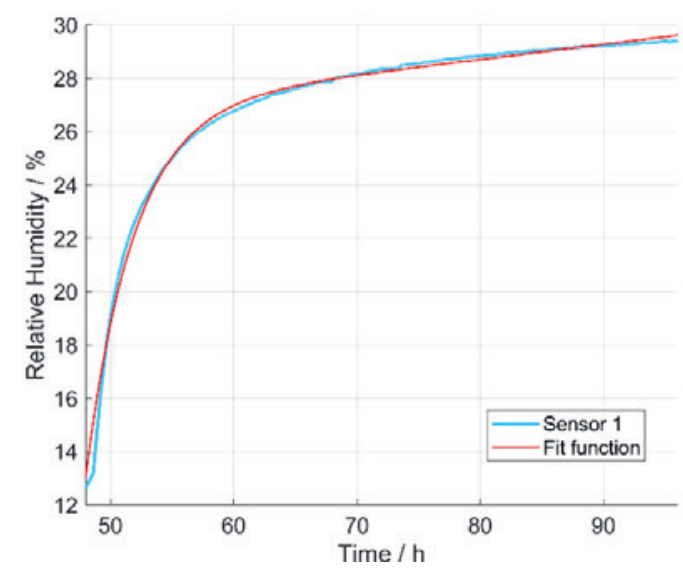

Fig. 3: Step from 10 to $30 \%$ r.H. for Sensor 1 with fitted function: $f(x)=24.57 \cdot e^{0.002 x}-3157234.10 \cdot e^{-0.26 x}$

Tab. 2: Sensor response time constants

\begin{tabular}{|c|c|}
\hline Sensor No. & Time to $\mathbf{3 0} \%$ r.H. t99 [h] \\
\hline 1 & 36.9 \\
\hline 2 & 70.3 \\
\hline 3 & 133.0 \\
\hline 4 & 210.3 \\
\hline
\end{tabular}

\section{Conclusion}

In this work, the entry process of moisture e.g. by diffusion through the silicone-potting compound SYLGARD-527 and/or passing along the boundary between glass and the silicone is investigated by using wireless humidity sensors potted inside the silicone. The sensors were inductively powered and read out via Bluetooth. This way, other possible moister gateways e.g. via cables were excluded. From the experimental results in becomes clear, that under experimental conditions that the moisture just diffuses through the silicone and does not pass along the boundary layer between the glass and the silicone. In order to transfer this result to the converters, we will use the same setup in a converter module housing and add additional moister gateways, as cables and printed circuit boards, to be presented at the conference.

\section{Acknowledgment}

Supported by the German Federal Ministry of Economics and Technology (BMWi) upon decision of the German Bundestag under the Grant 0324336E.

\section{References}

[1] S. Harley, E.Glascoe, R. Maxwell, Thermodynamic Study on Dynamic Water Vapor Sorption in Sylgard184, The Journal of Physical Chemistry B 116 (48), 14183-14190 (2012); doi: 10.1021/jp305997f

[2] C. Zorn, M. Piton, N. Kaminski, Impact of Humidity on Railway Converters, PCIM Europe 2017, Nürnberg, 715-722 (2017); ISBN: 978-3-8007-4424-4

[3] Sensirion AG, Datasheet SHT3x-DIS (2019) 